\title{
Investigação psicológica em contexto prisional português: uma revisão sistemática da literatura e desafios futuros para a investigação
}

\author{
Vítor Costa ${ }^{1}$, Samuel Monteiro ${ }^{1,2}$, Graça Esgalhado ${ }^{1,3}$ \& Henrique Pereira ${ }^{1,4}$ \\ ${ }^{1}$ Universidade da Beira Interior; BSAFE LAB Law Enforcement, Justice and Public Safety Research and \\ Technology Transfer Laboratory, Covilhã, Portugal \\ ${ }^{2}$ NECE - Research Center in Business Sciences, Covilhã, Portugal \\ ${ }^{3}$ IPCDHS - Instituto de Psicologia Cognitiva, Desenvolvimento Humano e Social, Coimbra, Portugal \\ ${ }^{4}$ Centro de Investigação em Ciências da Saúde, Covilhã, Portugal
}

\begin{abstract}
Resumo: 0 presente artigo procura, por meio de uma revisão sistemática da literatura, identificar e compreender as temáticas predominantes na investigação em contexto prisional português, identificando oportunidades de investigação futura. A análise de 41 artigos permite identificar 6 tipologias de estudos que caracterizam este domínio de investigação, nomeadamente, estudos empíricos com população adulta, população juvenil e com staff prisional, estudos de adaptação e/ou validação de instrumentos, programas de intervenção e artigos teóricos. A nível temático, a investigação com população forense adulta, que representa 18 dos 41 estudos analisados, centra-se nos domínios da psicopatologia e perturbações de personalidade. Os resultados permitem concluir que a investigação psicológica em contexto prisional se tem centrado, maioritariamente, em temas psicológicos clássicos, do domínio clínico, com recolha de dados junto dos reclusos. A utilização de abordagens multinível e a investigação nos domínios organizacional, ocupacional, educacional e da sexualidade humana, são identificados como oportunidades de investigação futura.
\end{abstract}

Palavras-chave: Investigação; Psicologia; Perturbações de personalidade; Psicopatia; Psicopatologia; Prisões; Revisão sistemática da literatura.

Psychological research in the Portuguese prison context: a systematic literature review and challenges for future research: The present paper aims, through a systematic literature review, at identifying and understanding the main themes in the Portuguese research in the prison context, identifying future research opportunities. The analysis of 41 papers allow the authors to identify 6 typologies of studies in this field, namely, empirical studies with adult samples, juvenile samples and prison staff, studies that work on the adaptation and validation of measures, intervention programs and theoretical papers. Thematically, the research with adult forensic populations, which represents 18 out of 41 papers, focuses on the psychopathology and personality disorders domains. The results allow the conclusion that psychological research in the prison context has focused in classic psychological themes, from the clinical domain, collecting data from inmates. The authors identify the use of multilevel approaches and research in the organizational, occupational, educational and human sexuality domains as opportunities for future research.

Keywords: Research; Psychology; Personality disorders; Psychopathology; Prisons; Systematic literature review.

A investigação psicológica, em contexto prisional, realizada em Portugal, tem gerado um elevado número de publicações que carecem de uma análise estruturada, de forma a sistematizar as suas principais contribuições para a investigação e prática de avaliação e intervenção em psicologia. 0 contexto prisional, apresenta-se como um local privilegiado para a investigação e prática da psicologia, uma vez que abrange domínios, necessidades e problemáticas não apenas das três especialidades clássicas da psicologia, reconhecidas pela ordem dos psicólogos portugueses, mas também outros domínios de especialidade avançada, constituindo-se como um sistema que promove a multiplicidade de estudos e investigações, que reclamam, atualmente, uma análise integrada e uma revisão de amplo espectro. 0 presente estudo pretende, assim, por meio de uma revisão sistemática da literatura (RSL), analisar contribuições teóricas

${ }^{1}$ Endereço para correspondência: Vítor Costa, Departamento de Psicologia e Educação, Estrada do Sineiro, s/n, 6200-209 Covilhã. E-mail: vitormvc@ubi.pt. 
(de autores portugueses ou associados a instituições de ensino portuguesas) e empíricas (com dados recolhidos no sistema prisional português) publicadas em revistas indexadas em duas reconhecidas bases de dados (Web of Science e SCOPUS). Com esta revisão, os autores pretendem identificar e compreender quais as temáticas predominantes na investigação em contexto prisional português, facultando um referencial atualizado do estado da arte - resultante de uma abordagem metodológica com mais-valias face a uma mera revisão narrativa, bem como, pretendem identificar e projetar oportunidades de investigação futura no contexto em estudo.

0 artigo encontra-se estruturado da seguinte forma: a secção 2 apresenta a componente metodológica desta revisão, num processo desde as palavras-chave até ao número final de artigos incorporados no presente trabalho; a secção 3 apresenta os resultados, onde os artigos considerados são divididos em 6 grupos distintos; a secção 4 apresenta as conclusões, discute os resultados obtidos e as suas principais limitações, apresentando ainda as oportunidades identificadas de investigação futura.

\section{METODOLOGIA}

A presente revisão da literatura segue linhas orientadoras internacionais, provenientes das ciências médicas (Moher, Liberati, Tetzlaff, \& Altman, 2009) e das ciências sociais (Denyer \& Tranfield, 2009; Saur-Amaral, 2011). As palavras-chave utilizadas nas bases de dados, selecionadas após pesquisas exploratórias, foram: "inmate*", "prison*" e "offender". Com recurso à linguagem boolean, as diferentes palavras-chave podem aparecer de forma isolada nos artigos, isto é, foi utilizada a conjunção "ou” (OR). Adicionalmente, e em concordância com os objetivos do presente estudo, foram filtrados os resultados de acordo com o domínio científico (psicologia) e país (Portugal). As pesquisas realizadas, que terminaram em dezembro de 2015, contemplam artigos publicados nas bases de dados até novembro de 2015. Após eliminados os resultados duplicados, foram obtidas 110 referências. Após análise do título e resumo, e aplicação dos critérios de inclusão e exclusão (cf. Anexo I), 46 artigos foram selecionados para a presente revisão, estando disponível o acesso integral a 41 artigos. A figura 1 resume o processo de pesquisa e acesso às referências.



Figura 1. Diagrama com o processo de identificação, seleção e inclusão de referências.

\section{RESULTADOS}

Os artigos considerados na presente RSL foram inicialmente divididos em diferentes categorias, de forma a facilitar a análise comparativa dos mesmos. Dos 41 artigos que totalizam a amostra, foram criados grupos distintos com base em critérios amostrais e de tipologia do artigo, resultando em 6 grupos: (1) estudos empíricos com população forense adulta $(n=18)$; (2) estudos empíricos com população forense juvenil ( $n=5$ ); (3) estudos de adaptação e/ou validação de instrumentos $(n=6)$; (4) Programas de intervenção $(n=4)$; (5) artigos teóricos $(n=6)$ e (6) artigos empíricos envolvendo staff prisional $(n=2)$. A análise dos artigos encontra-se assim segmentada de acordo com os grupos identificados.

\section{Estudos empíricos com população forense adulta}

Os estudos empíricos com população forense adulta, com 18 dos 41 artigos considerados, representam $44 \%$ dos artigos analisados. Assim sendo, realiza-se uma análise mais pormenorizada dos referidos 
estudos, avaliando-se características da amostra dos estudos e realizando-se uma análise temática aos mesmos.

\section{Amostra}

Ao nível da amostra, é de referir que a grande maioria dos estudos (15 em 18) recolheu dados com população do género masculino, sendo que apenas dois recolheram dados junto de reclusos dos dois géneros (Alves, Dutra, \& Maia, 2013; Fonseca, Neto, \& Mullet, 2012) e um estudo recolheu exclusivamente dados em população feminina (Alves \& Maia, 2010). Uma outra tendência verificada é a de selecionar uma população para recolha de dados, de acordo com características dos reclusos, tais como: (1) reincidência (Barbosa \& Monteiro, 2008; Seruca \& Silva, 2015); (2) tipo de crime, como por exemplo, crimes sexuais (Pechorro, Poiares, \& Vieira, 2008; Rebocho \& Gonçalves, 2012; Sigre-Leirós, Carvalho, \& Nobre, 2015a, 2015b), violência doméstica (Cunha \& Gonçalves, 2013), ou crimes contra o património (Fonseca, 2014) e (3) fase de execução da pena (Moreira \& Gonçalves, 2010). Em termos de dimensão, as amostras forenses dos estudos analisados são tendencialmente pequenas, considerando que apenas 4 dos 18 estudos recolheu dados em mais de 100 reclusos (Brazão, da Motta, Rijo, \& Pinto-Gouveia, 2015; Cunha \& Gonçalves, 2013; Gonçalves, 1999; Rebocho \& Gonçalves, 2012).

\section{Análise Temática}

Considerando o número de artigos que recolheu dados junto de população adulta forense, foi realizada uma análise temática dos mesmos, através dos objetivos dos estudos, seguindo um procedimento de codificação aberta (Corbin \& Strauss, 1990), que permitiu a organização dos artigos em 5 temáticas:

1. Perturbações de personalidade: este primeiro tema agrega 6 artigos nos quais a avaliação da personalidade é um dos objetivos centrais. Considerando o contexto (forense) dos estudos em análise, 3 artigos debruçam-se, especificamente, sobre a psicopatia (perturbação antissocial da personalidade). Esta perturbação foi, nestes 3 artigos, avaliada com recurso ao Hare Psychopathy Checklist-Revised (PCL-R) (Cunha \& Gonçalves, 2013; Gonçalves, 1999; Iria \& Barbosa, 2009). Os restantes estudos, optando por uma avaliação não exclusiva da psicopatia, avaliaram as perturbações de personalidade com recurso ao Inventário Clínico Multiaxial de Millon-II (MCMI-II) (Duque \& Neves, 2004; Pechorro et al., 2008), e com recurso a entrevista clínica estruturada (Brazão et al., 2015).

2. Psicopatologia: neste segundo tema, encontramos 5 artigos que remetem para a avaliação de sintomas psicopatológicos, o que aconteceu, na totalidade dos artigos, mediante a utilização do inventário de sintomas psicopatológicos (BSI) (Alves et al., 2013; Alves \& Maia, 2010; Moreira \& Gonçalves, 2010; Sigre-Leirós et al., 2015b).

3. Emoções: dois estudos centram-se na avaliação de respostas emocionais como o ressentimento e perdão (Fonseca et al., 2012; Fonseca, 2014).

4. Funções executivas: dois estudos seguem uma abordagem neuropsicológica avaliando o funcionamento pré-frontal em diferentes grupos (Barbosa \& Monteiro, 2008; Seruca \& Silva, 2015).

5. Questões criminológicas e forenses: por último, um conjunto de 3 estudos debruça-se sobre o estudo de questões como a adaptação à prisão, sugestionabilidade interrogativa e comportamento criminal (Gonçalves \& Gonçalves, 2012; Penajoia, Pinho, \& Dias, 2015; Rebocho \& Gonçalves, 2012).

\section{Estudos empíricos com população forense juvenil}

Cinco estudos apresentam uma análise realizada com dados recolhidos junto de jovens institucionalizados. Deste total, 4 têm como foco a temática da psicopatia, procurando verificar a influência desta variável ao nível da idade de início da atividade criminal em mulheres (Pechorro, Gonçalves, Maroco, Nunes, \& Jesus, 2014) e homens (Pechorro, Maroco, Gonçalves, Nunes, \& Jesus, 2014), bem como analisando diferenças de género (Pechorro, Poiares, Marôco, \& Vieira, 2012) e étnicas (Pechorro, Poiares, Barroso, Nunes, \& Jesus, 2015). Já o estudo de Vilariño, Amado e Alves (2013), realizado com 84 adolescentes (42 dos quais em centro educativo), conclui que os jovens institucionalizados apresentam problemas ao nível da socialização (consideração pelos outros, isolamento social e ansiedade social/timidez), assim como défice de inteligência emocional (nomeadamente na dimensão clareza de sentimentos), empregando estratégias de coping desajustadas (e.g., ignorar problema, autoincriminar-se).

\section{Estudos de adaptação e/ou validação de instrumentos}

Seis artigos apresentam um trabalho de adaptação e/ou validação de instrumentos para o contexto forense português. A Tabela 1 apresenta as características da amostra, instrumento analisado e 
dimensionalidade do mesmo, bem como informações adicionais quanto ao número de itens e escala de resposta.

Tabela 1. Estudos de adaptação e/ou validação de instrumentos

\begin{tabular}{|c|c|c|c|}
\hline Autor & Amostra & $\begin{array}{l}\text { Instrumento } \\
\text { adaptado/validado }\end{array}$ & O que avalia; Informações adicionais \\
\hline (Talina et al., 2013) & $\begin{array}{l}143 \text { indivíduos } \\
\text { adultos em contexto } \\
\text { psiquiátrico forense. }\end{array}$ & $\begin{array}{l}\text { CANFOR (The } \\
\text { Camberwell } \\
\text { Assessment of Need } \\
\text { - Forensic Version) }\end{array}$ & $\begin{array}{l}\text { Avalia as necessidades dos utentes em contextos de } \\
\text { saúde mental forense. Considera } 25 \text { domínios, que são } \\
\text { cotados como ausentes ou presentes. }\end{array}$ \\
\hline $\begin{array}{l}\text { (Pechorro, Maroco, } \\
\text { Poiares, \& Vieira, } \\
2011 \text { ) }\end{array}$ & $\begin{array}{l}250 \text { jovens (entre } 13 \\
\text { e } 20 \text { anos), reclusos } \\
\text { em centros de } \\
\text { detenção juvenis e } \\
510 \text { jovens (entre } 12 \\
\text { e } 20 \text { anos) de } \\
\text { amostra não forense. }\end{array}$ & $\begin{array}{l}\text { Antisocial Process } \\
\text { Screening Device- } \\
\text { Self-Report (APSD- } \\
\text { SR) }\end{array}$ & $\begin{array}{l}\text { Avalia traços psicopáticos através de um instrumento de } \\
\text { autorresposta de } 20 \text { itens numa escala ordinal de } 3 \\
\text { pontos. A versão portuguesa encontra suporte para uma } \\
\text { estrutura bidimensional: (1) fraco controlo dos } \\
\text { impulsos/problemas comportamentais e (2) } \\
\text { insensível/sem emoção. }\end{array}$ \\
\hline $\begin{array}{l}\text { (Pechorro, Barroso, } \\
\text { Maroco, Vieira, \& } \\
\text { Gonçalves, 2015) }\end{array}$ & $\begin{array}{l}192 \text { participantes } \\
\text { homens, de } 8 \\
\text { centros de detenção } \\
\text { juvenil. }\end{array}$ & $\begin{array}{l}\text { Psychopathy } \\
\text { Checklist: Youth } \\
\text { Version (PCL:YV) }\end{array}$ & $\begin{array}{l}\text { Avalia características comportamentais e de } \\
\text { personalidade associadas com a psicopatia, utilizando } 20 \\
\text { itens, numa escala ordinal de } 3 \text { pontos. Pode ser obtida } \\
\text { uma pontuação total. Os resultados do estudo apontam } \\
\text { para uma organização e } 16 \text { itens em } 3 \text { fatores } \\
\text { (interpessoal; afetivo; comportamental). }\end{array}$ \\
\hline $\begin{array}{l}\text { (Pechorro, Maroco, } \\
\text { Ray, \& Gonçalves, } \\
2015 \text { ) }\end{array}$ & $\begin{array}{l}221 \text { homens em } \\
\text { centros de detenção } \\
\text { juvenil. }\end{array}$ & $\begin{array}{l}\text { Barratt } \\
\text { Impulsiveness Scale } \\
\text { version } 11 \text { (BIS-11) }\end{array}$ & $\begin{array}{l}\text { Mede a impulsividade, organizada em } 6 \text { fatores de } 1 .^{\underline{a}} \\
\text { ordem e } 3 \text { fatores de } 2 . \underline{\text { a }} \text { ordem (impulsividade } \\
\text { atencional, motora e não planeada), através de } 30 \text { itens } \\
\text { numa escala ordinal de } 4 \text { pontos. }\end{array}$ \\
\hline $\begin{array}{l}\text { (Pechorro, Ray, Salas- } \\
\text { Wright, Maroco, \& } \\
\text { Gonçalves, 2015) }\end{array}$ & $\begin{array}{l}221 \text { homens em } \\
\text { centros de detenção } \\
\text { juvenil. }\end{array}$ & $\begin{array}{l}\text { Basic Empathy Scale } \\
\text { (BES) }\end{array}$ & $\begin{array}{l}\text { Mede a empatia em adolescentes, utilizando uma escala } \\
\text { ordinal de } 5 \text { pontos e } 20 \text { itens agrupados em dois fatores } \\
\text { (empatia afetiva e empatia cognitiva), ou sete itens } \\
\text { agrupados em dois fatores, na versão curta. }\end{array}$ \\
\hline $\begin{array}{l}\text { (Pimentel, Quintas, } \\
\text { Fonseca, \& Serra, } \\
\text { 2015) }\end{array}$ & $\begin{array}{l}2363 \text { jovens em } \\
\text { contacto com a } \\
\text { DGRSP. }\end{array}$ & $\begin{array}{l}\text { Youth Level of } \\
\text { Service/Case } \\
\text { Management } \\
\text { Inventory } \\
\text { (YLS/CMI). }\end{array}$ & $\begin{array}{l}\text { O YLS/CMI é uma ferramenta de avaliação de } \\
\text { necessidades (criminogénicas) e riscos (de reincidência), } \\
\text { bem como de gestão de casos de jovens delinquentes. No } \\
\text { estudo foram utilizados } 42 \text { itens para avaliar } \\
\text { necessidades e riscos, codificados como presentes (1) ou } \\
\text { ausentes (0). }\end{array}$ \\
\hline
\end{tabular}

\section{Programas de intervenção}

Os programas de intervenção em contexto prisional são analisados por 4 artigos. Três dos quais remetem para o programa Gerar Percursos Sociais (GPS), apresentado no artigo de Brazão, da Motta e Rijo (2013). Este programa visa uma intervenção cognitivo-comportamental junto de reclusos, distinguindo-se assim das abordagens tradicionais à reabilitação, que apostavam numa componente psicoeducativa. Tem como objetivo a intervenção em comportamentos antissociais e a reabilitação de jovens delinquentes. Estruturado com base num referencial teórico que olha para a origem e manutenção do comportamento desviante numa perspetiva cognitivo-interpessoal, esta intervenção estrutura-se em 5 módulos, nomeadamente: (1) comunicação humana; (2) relações interpessoais; (3) distorções cognitivas; (4) funcionamento e significado das emoções; (5) crenças centrais disfuncionais. Os resultados desta intervenção, avaliados com pré e pós teste, num grupo que participou na intervenção (n=24) e num grupo de controlo $(n=24)$ revelam que a intervenção produz mudanças clínicas significativas ao nível emocional, isto é, na dimensão raiva (traço) e ainda ao nível cognitivo (paranóia) (Brazão et al., 2015b). Numa outra publicação, uma análise da covariância (ANCOVA) reforça a existência de diferenças estatisticamente significativas entre o grupo alvo de intervenção e grupo de controlo, bem como dentro do grupo de intervenção em momento pré e pós programa de intervenção, sustentando assim a adequabilidade do GPS na alteração de esquemas cognitivos que sustentam comportamentos antissociais (Brazão et al., 2015a). Por último, o estudo de Rocha, Marques, Fortuna, Antunes e Hoaken (2014) apresenta um programa de intervenção cognitiva, no qual participaram 28 mulheres reclusas, cada uma recebendo uma média de 28 sessões de intervenção (individuais). Uma análise pré e pós intervenção detetou diferenças positivas estatisticamente significativas em domínios como a atenção, rapidez de processamento, aprendizagem verbal, memória, flexibilidade cognitiva e planeamento, e negativas em estados emocionais como depressão, ansiedade, stress e comportamento perturbado em contexto prisional. 


\section{Contribuições teóricas}

O artigo teórico de Gonçalves (2007) expõe os desafios de avaliação e intervenção com ofensores conjugais e sexuais, com (ou sem) diagnóstico de psicopatia. Propõe procedimentos para a avaliação (do processo e dos resultados), seleção dos programas de intervenção e indivíduos a intervencionar, alertando ainda para a importância de staff altamente qualificado dadas as exigências do trabalho com este tipo de população, de forma a aumentar a eficácia e eficiência da avaliação e intervenção em personalidades do tipo antissocial. A avaliação e intervenção na psicopatia merecem a atenção de Moreira, Almeida, Pinto e Fávero (2014), que refletem sobre a complexidade da conceptualização e avaliação da psicopatia. Apresentando a psicopatia, enquanto um constructo presente em toda a população, defendem que será mais apropriado falar de pessoas com fortes características de perturbação antissocial da personalidade, ao invés da presença (ou ausência) de psicopatia. As dificuldades de avaliação da psicopatia através de questionários de autorresposta, bem como a necessidade de desenvolvimento de programas de intervenção específicos para esta população (uma vez que as intervenções tradicionais são consideradas como ineficientes ou mesmo contraproducentes), marcam ainda este contributo teórico.

Na meta análise realizada por Gonçalves, Gonçalves, Martins e Dirkzwager (2014). Por completa, foram analisados 90 estudos em torno de dois preditores de ajustamento ao contexto prisional: as infrações institucionais e a utilização dos serviços de saúde. Estes preditores foram depois agrupados em individuais (e.g., agressividade, impulsividade) e contextuais (e.g., atividade de gangs, prisões com mais reclusos e de maior segurança). Os autores detetam a necessidade de investigar as variáveis contextuais (e.g., sobrelotação, nível de segurança, dimensão, rácio staff-reclusos, proporção de reclusos com atividade laboral), enquanto preditores, e a utilização de serviços de saúde, enquanto resultado, do ajustamento dos reclusos ao meio prisional. 0 artigo de Silva, Rijo e Salekin (2015) apresenta-nos a psicopatia numa perspetiva da psicologia evolutiva, ou seja, a psicopatia não é compreendida exclusivamente como uma psicopatologia, mas também como uma estratégia adaptativa de lidar com ambientes psicológicos hostis. 0 contributo das teorias evolutivas para a compreensão das origens e da trajetória desenvolvimental de indivíduos com traços psicopáticos é também abordado numa outra contribuição de Silva, Rijo e Salekin (2012), que se debruça sobre a etiologia da psicopatia e modelos preditores da agressividade e comportamento violento, de formar a potenciar a intervenção efetiva tanto ao nível terapêutico como ao nível preventivo.

Fora da temática da psicopatia, o artigo de Rebocho e Silva (2014) revê a literatura no âmbito do comportamento predatório (hunting behavior) e seleção de alvos de violadores, seguindo um ponto de vista da criminologia ambiental, na qual o ofensor é apenas mais um elemento do crime, sendo também estudada a localização dos crimes, procurando-se assim padrões sobre como, onde e como ocorrem os crimes.

\section{Estudos envolvendo staff prisional}

No âmbito dos artigos considerados, apenas dois estudos recolheram dados junto de staff prisional (Gonçalo, Gomes, Barbosa, \& Afonso, 2010; Mendes, Claro, \& Robazzi, 2014). Inquirindo 95 profissionais de enfermagem de 7 estabelecimentos prisionais diferentes, o estudo descritivo de Mendes et al. (2014) avaliou o burnout, com recurso ao MBI-GS, concluindo que 31,57\% dos respondentes apresentam níveis de burnout nas dimensões de exaustão e cinismo e 6,32\% na dimensão de eficácia profissional. Os profissionais com contrato de trabalho com o ministério da justiça apresentaram níveis significativamente superiores aos profissionais em regime de prestação de serviço. Utilizando o mesmo instrumento de avaliação do burnout, o estudo de Gonçalo et al. (2010) comparou 237 guardas prisionais com 95 trabalhadores das forças de segurança pública. Os resultados evidenciaram diferenças estatisticamente significativas ao nível da exaustão emocional e cinismo (maior nas forças de segurança prisionais), bem como no comprometimento organizacional, satisfação com a vida, satisfação profissional (menor nas forças de segurança prisionais) e ainda um maior desejo de abandonar a profissão nos profissionais em contexto prisional.

\section{CONSIDERAÇõES FINAIS E IMPLICAÇõES PARA A INVESTIGAÇÃO FUTURA}

Os resultados apresentados nesta revisão permitem-nos concluir que a investigação psicológica em contexto prisional se tem centrado, maioritariamente, em temas psicológicos clássicos, do domínio clínico (psicopatologia e perturbações de personalidade), com recolha de dados junto dos reclusos. Revelam ainda um esforço recente na adaptação de medidas para avaliação de variáveis psicológicas em contexto prisional, bem como na avaliação da eficácia de programas de intervenção (um caminho importante na afirmação e legitimação do trabalho do psicólogo em contexto prisional). No entanto, esta orientação 
maioritária da investigação para uma abordagem clínica reflete-se na escassez de estudos com staff prisional (apenas 2 em 41), remetendo-nos para uma leitura das variáveis psicológicas em contexto prisional maioritariamente centradas em dados obtidos com reclusos. Os reclusos investigados têm sido maioritariamente do género masculino, o que espelha as características da população reclusa, sendo as amostras pequenas, o que poderá refletir as dificuldades e restrições associadas à recolha de dados num contexto desta natureza.

0 trabalho desenvolvido, com base na metodologia da revisão sistemática da literatura, apresenta um contributo com diversas implicações e mais-valias, tais como: (1) metodologia passível de ser replicada; (2) análise conjunta de um número diverso de contribuições teóricas e empíricas em contexto prisional português; (3) estado da arte que permite uma análise atual das possibilidades de investigação futura em contexto prisional português. No entanto, a presente revisão não está isenta de limitações. Embora tenha existido um esforço exaustivo de análise de artigos pertencentes a duas bases de dados de reconhecida importância (Web of Science e SCOPUS), material não publicado e publicações de outra natureza (como dissertações de mestrado) não foram consideradas na presente análise. Adicionalmente, refere-se que o tipo de pesquisa e filtros utilizados podem estar sujeitos a imprecisões nos automatismos e estão dependentes da catalogação dos artigos nas bases de dados.

A realização da presente revisão permite uma sistematização exaustiva e alargada da investigação psicológica realizada em contexto prisional português, publicada em revistas científicas indexadas na Web of Science e SCOPUS. Os resultados apresentados indicam-nos, no entanto, diversas possibilidades de investigação futura, essencialmente quando confrontamos os resultados atuais com investigação realizada em outros países. Nesse sentido, emergem como possibilidades de investigação futura:

1. Abordagens multinível: a complexidade do estudo de variáveis psicológicas em contexto prisional poderá ser acompanhada de abordagens metodológicas que representam essa mesma complexidade. Nesse sentido, o estudo do comportamento humano em contexto prisional poderá incorporar, através da recolha e análise de dados em múltiplos níveis de análise, a relação com variáveis de nível grupal ou organizacional (e.g., Camp, Gaes, Langan, \& Saylor, 2003; Camp \& Gaes, 2005; Lahm, 2007; Molleman \& van der Broek, 2014; Molleman \& van Ginneken, 2015; Molleman \& Leeuw, 2012; Morris \& Worrall, 2010; Wooldredge, Griffin, \& Pratt, 2001).

2. Ramos da psicologia: estando a investigação existente centrada, maioritariamente, no nível individual, e nos reclusos enquanto público-alvo, consideramos que carece de investigação, em contexto prisional, temas de âmbito organizacional, ocupacional, educacional e da sexualidade humana. Nomeadamente, é escassa a investigação desenvolvida com staff prisional, apesar de reconhecido que este grupo se encontra exposto a elevados riscos psicossociais no seu contexto de trabalho. Desse modo, o estudo nesta população de temas como o balanço e conflito trabalho-família (Lambert, Hogan, \& Barton, 2002; Tavares, Caetano, \& Silva, 2007; Triplett, Mullings, \& Scarborough, 1999), os riscos psicossociais (Ghaddar, Ronda, Nolasco, Álvares, \& Mateo, 2011; Ghaddar, Ronda, \& Nolasco, 2011) e as condições físicas do estabelecimento prisional (Bierie, 2012) ganham uma importância acrescida, considerando os níveis de burnout identificados em investigação anterior (Gonçalo et al., 2010; Mendes et al., 2014) e o risco de suicídio destes profissionais (Stack \& Tsoudis, 1997). Outras temáticas como a sobrelotação (Lawrence \& Andrews, 2004; Martin, Lichtenstein, Jenkot, \& Forde, 2012; Sharkey, 2010) e a qualidade de vida em contexto prisional (Crewe, Liebling, \& Hulley, 2011; Liebling, 2008) carecem ainda de investigação em contexto português. Também a sexualidade humana em geral, e a saúde sexual em particular, se apresentam como importantes tópicos a explorar futuramente. Embora se registe investigação neste domínio em contexto português (Coelho \& Gonçalves, 2010), escasseia ainda o conhecimento no âmbito dos comportamentos e saúde sexual em contexto prisional, sendo esta necessidade generalizável a outros países (cf. Stewart, 2007), facto que poderá ser explicado não tanto pelo interesse ou desinteresse da temática mas principalmente pelos obstáculos encontrados no processo de recolha de dados (cf. Barth, 2012).

3. Outros temas emergentes: a ameaça terrorista tem levado nos últimos anos a uma atenção crescente para os fenómenos de radicalização, na sociedade em geral, mas também em contexto prisional (Awan, 2013; Ganor \& Falk, 2013; Jones, 2014; Khosrokhavar, 2013; Mulcahy, Merrington, \& Bell, 2013; Trujillo, Jordán, Antonio Gutiérrez, \& González-Cabrera, 2009; Useem \& Clayton, 2009). Nesse sentido, existe na atualidade uma procura crescente por instrumentos de medida da vulnerabilidade e risco de radicalização, bem como programas de intervenção no âmbito da desradicalização, onde o papel do psicólogo, no contexto de equipas multidisciplinares, será fundamental, considerando-se, assim, esta como uma área emergente da atuação do psicólogo em contexto prisional. 


\section{Referências}

Alves, J., Dutra, A., \& Maia, Â. (2013). História de adversidade, saúde e psicopatologia em reclusos: comparação entre homens e mulheres. Ciência \& Saúde Coletiva, 18(3), 701-709. http://doi.org/10.1590/S1413-81232013000300016

Alves, J., \& Maia, Â. (2010). Experiências adversas durante a infância e comportamentos de risco para a Saúde em mulheres reclusas. Psicologia, Saúde E Doenças, 11(1), 151-171.

Awan, I. (2013). Muslim Prisoners, Radicalization and Rehabilitation in British Prisons. Journal of Muslim Minority Affairs, 33(3), 371-384. http://doi.org/10.1080/13602004.2013.853979

Barbosa, M., \& Monteiro, L. (2008). Recurrent criminal Behavior and executive dysfunction. Spanish Journal of Psychology, 11(1), 259-265. https://doi.org/10.1017/S1138741600004297

Barth, T. (2012). Relationships and sexuality of imprisoned men in the German penal system-a survey of inmates in a Berlin prison. International Journal of Law and Psychiatry, 35(3), 153-158. http://doi.org/10.1016/j.ijlp.2012.02.001

Bierie, D. M. (2012). The Impact of Prison Conditions on Staff Well-Being. International Journal of Offender Therapy and Comparative Criminology, 56(1), 81-95. http://doi.org/10.1177/0306624x10388383

Brazão, N., da Motta, C., \& Rijo, D. (2013). From multimodal programs to a new cognitive-interpersonal approach in the rehabilitation of offenders. Aggression \& Violent Behavior, 18(6), 636-643. https://doi.org/10.1016/j.avb.2013.07.018

Brazão, N., da Motta, C., Rijo, D., \& Pinto-Gouveia, J. (2015). The prevalence of personality disorders in Portuguese male prison inmates: Implications for penitentiary treatment. Análise Psicológica, 33(3), 279-289. http://doi.org/10.14417/ap.975

Brazão, N., da Motta, C., Rijo, D., Salvador, M. do C., Pinto-Gouveia, J. J., \& Ramos, J. (2015a). Clinical Change in Cognitive Distortions and Core Schemas After a Cognitive-Behavioral Group Intervention: Preliminary Findings from a Randomized Trial with Male Prison Inmates. Cognitive Therapy \& Research, 39(5), 578-589. http://doi.org/10.1007/s10608-015-9693-5

Brazão, N., da Motta, C., Rijo, D., Salvador, M. do C., Pinto-Gouveia, J. J., \& Ramos, J. J. (2015b). Clinical change in anger, shame, and paranoia after a structured cognitive-behavioral group program: Early findings from a randomized trial with male prison inmates. Journal of Experimental Criminology, 11(2), 217-236. http://doi.org/10.1007/s11292-014-9224-5

Camp, S. D., \& Gaes, G. (2005). Criminogenic effects of the prison environment on inmate behavior: Some experimental evidence. Crime \& Delinquency. 51(3), 425-442. https://doi.org/10.1177/0011128704271471

Camp, S. D., Gaes, G. G., Langan, N. P., \& Saylor, W. G. (2003). The influence of prisons on inmate misconduct: A multilevel investigation. Justice Quarterly, 20(3), 501-533. http://doi.org/10.1080/07418820300095601

Coelho, C., \& Gonçalves, R. A. (2010). Prison Guards' Attitudes Towards the Prevention of Sexual Contacts Between Inmates. Howard Journal of Criminal Justice, 49(4), 361-374. http://doi.org/10.1111/j.1468-2311.2010.00626.x

Corbin, J., \& Strauss, A. (1990). Grounded theory research: Procedures, canons, and evaluative criteria. Qualitative Sociology. Zeitschrift für Soziologie, 19(6), 418-427. https://doi.org/10.1515/zfsoz1990-0602

Crewe, B., Liebling, A., \& Hulley, S. (2011). Staff culture, use of authority and prisoner quality of life in public and private sector prisons. Australian \& New Zealand Journal of Criminology, 44(1), 94-115. http://doi.org/10.1177/0004865810392681

Cunha, O. S., \& Gonçalves, R. A. (2013). Intimate partner violence offenders: Generating a data-based typology of batterers and implications for treatment. European Journal of Psychology Applied to Legal Context, 5(2), 131-139. http://doi.org/10.5093/ejpalc2013a2

Denyer, D., \& Tranfield, D. (2009). Producing a Systematic Review. In The SAGE Handbook of Organizational Research Methods (pp. 671-689). London: SAGE Publications Ltd.

Duque, A. F., \& Neves, P. G. (2004). Auto-mutilação em meio prisional: avaliação das perturbações da personalidade. Psicologia, Saúde E Doenças, 5(2), 215-227.

Fonseca, A. C. M. (2014). Resentment, forgiveness and revenge among violent people incarcerated for property offenses. Psicologica, 35(3), 613-620.

Fonseca, A. C. M., Neto, F. F., \& Mullet, E. (2012). Dispositional forgiveness among homicide offenders. Journal of Forensic Psychiatry \& Psychology, 23(3), 410-416. http://doi.org/10.1080/14789949.2012.668213

Ganor, B., \& Falk, O. (2013). De-Radicalization in Israel's Prison System. Studies in Conflict \& Terrorism, 36(2), 116-131. http://doi.org/10.1080/1057610X.2013.747071

Ghaddar, A., Ronda, E., \& Nolasco, A. (2011). Work ability, psychosocial hazards and work experience in 
prison environments. Occupational $\quad$ Medicine, 61(7), 503-508. http://doi.org/10.1093/occmed/kqr124

Ghaddar, A., Ronda, E., Nolasco, A., Álvares, N., \& Mateo, I. (2011). Exposure to psychosocial risks at work in prisons: Does contact with inmates matter? A pilot study among prison workers in Spain. Stress and Health, 27(2), 170-176. http://doi.org/10.1002/smi.1322

Gonçalo, H., Gomes, A. R., Barbosa, F., \& Afonso, J. (2010). Stresse ocupacional em forças de segurança: Um estudo comparativo. Análise Psicológica, 28(1), 165-178. http://doi.org/10.14417/ap.262

Gonçalves, L. C., \& Gonçalves, R. A. (2012). Agressividade, estilo de vida criminal e adaptação à prisão. Psicologia USP, 23(ahead), 0-0. http://doi.org/10.1590/S0103-65642012005000013

Gonçalves, L. C., Gonçalves, R. A., Martins, C., \& Dirkzwager, A. J. E. (2014). Predicting infractions and health care utilization in prison: A meta-analysis. Criminal Justice and Behavior, 41(8), 921-942. http://doi.org/10.1177/0093854814524402

Gonçalves, R. A. (1999). Psychopathy and offender types: Results from a Portuguese prison sample. International Journal of Law and Psychiatry, 22(3-4), 337-346. http://doi.org/10.1016/S01602527(99)00013-8

Gonçalves, R. A. (2007). Promover a mudança em personalidades anti-sociais: Punir, tratar e controlar. Análise Psicológica, 25(4), 571-583. http://doi.org/10.14417/ap.466

Iria, C., \& Barbosa, F. (2009). Perception of facial expressions of fear: Comparative research with criminal and non-criminal psychopaths. Journal of Forensic Psychiatry and Psychology, 20(1), 66-73. http://doi.org/10.1080/14789940802214218

Jones, C. R. (2014). Are prisons really schools for terrorism? Challenging the rhetoric on prison radicalization. Punishment \& Society, 16(1), 74-103. http://doi.org/10.1177/1462474513506482

Khosrokhavar, F. (2013). Radicalization in Prison: The French Case. Politics, Religion \& Ideology, 14(2), 284-306. http://doi.org/10.1080/21567689.2013.792654

Lahm, K. F. (2007). Inmate-On-Inmate Assault: A Multilevel Examination of Prison Violence. Criminal Justice and Behavior, 35(1), 120-137. http://doi.org/10.1177/0093854807308730

Lambert, E. G., Hogan, N. L., \& Barton, S. M. (2002). The Impact of Work-Family Conflict on Correctional Staff Job Satisfaction: An Exploratory Study. American Journal of Criminal Justice, 27(1), 35-52. http://doi.org/10.1007/BF02898969

Lawrence, C., \& Andrews, K. (2004). The influence of perceived prison crowding on male inmates' perception of aggressive events. Aggressive Behavior, 30(4), 273-283. http://doi.org/10.1002/ab.20024

Liebling, A. (2008). Incentives and Earned Privileges Revisited: Fairness, Discretion, and the Quality of Prison Life. Journal of Scandinavian Studies in Criminology and Crime Prevention, 9(February 2014), 25-41. http://doi.org/10.1080/14043850802450773

Martin, J. L., Lichtenstein, B., Jenkot, R. B., \& Forde, D. R. (2012). “They Can Take Us Over Any Time They Want": Correctional Officers' Responses to Prison Crowding. The Prison Journal, 92(1), 88-105. http://doi.org/10.1177/0032885511429256

Mendes, A., Claro, M., \& Robazzi, M. (2014). Burnout in nurses working in Portuguese central prisons and type of employment contract. Medicina Del Lavoro, 105(3), 214-222.

Moher, D., Liberati, A., Tetzlaff, J., \& Altman, D. (2009). Preffered reporting Items for Systematic and MetaAnalyses: the PRISMA Statement. PLOS Medicine, 6(7), e1000097. https://doi.org/10.1371/journal.pmed.1000097

Molleman, T., \& Leeuw, F. L. (2012). The Influence of Prison Staff on Inmate Conditions: A Multilevel Approach to Staff and Inmate Surveys. European Journal on Criminal Policy and Research, 18, 217233. http://doi.org/10.1007/s10610-011-9158-7

Molleman, T., \& van der Broek, T. C. (2014). Understanding the links between perceived prison conditions and prison staff. International Journal of Law, Crime and Justice, 42(1), 33-53. http://doi.org/10.1016/j.ijlcj.2014.01.001

Molleman, T., \& van Ginneken, E. F. J. C. (2015). A Multilevel Analysis of the Relationship Between Cell Sharing, Staff-Prisoner Relationships, and Prisoners' Perceptions of Prison Quality. International Journal of Offender Therapy and Comparative Criminology, 59(10), 1029-1046. http://doi.org/10.1177/0306624X14525912

Moreira, D., Almeida, F., Pinto, M., \& Fávero, M. (2014). Psychopathy: A comprehensive review of its assessment and intervention. Aggression and Violent Behavior, 19(3), 191-195. http://doi.org/10.1016/j.avb.2014.04.008

Moreira, N. A. C., \& Gonçalves, R. A. (2010). Perturbação mental e ideação suicida entre reclusos preventivos. Análise Psicológica, 28(1), 133-148. http://doi.org/10.14417/ap.260

Morris, R. G., \& Worrall, J. L. (2010). Prison Architecture and Inmate Misconduct: A Multilevel Assessment. 
Crime \& Delinquency, 60(7), 1083-1109. http://doi.org/10.1177/0011128710386204

Mulcahy, E., Merrington, S., \& Bell, P. J. (2013). The Radicalisation of Prison Inmates: A Review of the Literature on Recruitment, Religion and Prisoner Vulnerability. Journal of Human Security, 9(1), 414. http://doi.org/10.12924/johs2013.09010004

Pechorro, P., Barroso, R., Maroco, J., Vieira, R. X., \& Gonçalves, R. A. (2015). Psychometric Properties of the Psychopathy Checklist: Youth Version Among Portuguese Juvenile Delinquents. International Journal of Offender Therapy and Comparative Criminology, 59(12), 1322-1337. http://doi.org/10.1177/0306624x14535558

Pechorro, P., Gonçalves, R. A., Maroco, J., Nunes, C., \& Jesus, S. N. (2014). Age of Crime Onset and Psychopathic Traits in Female Juvenile Delinquents. International Journal of Offender Therapy and Comparative Criminology, 58(9), 1101-1119. http://doi.org/10.1177/0306624x13489864

Pechorro, P., Maroco, J., Gonçalves, R. A., Nunes, C., \& Jesus, S. N. (2014). Psychopathic traits and age of crime onset in male juvenile delinquents. European Journal of Criminology, 11(3), 288-302. http://doi.org/10.1177/1477370813495759

Pechorro, P., Maroco, J., Poiares, C., \& Vieira, R. X. (2011). Validation of the Portuguese Version of the Antisocial Process Screening Device-Self-Report With a Focus on Delinquent Behavior and Behavior Problems. International Journal of Offender Therapy and Comparative Criminology, 57(2), 112-126. http://doi.org/10.1177/0306624X11427174

Pechorro, P., Maroco, J., Ray, J. V, \& Gonçalves, R. A. (2015). Psychometric properties of the Barratt Impulsiveness Scale version 11 among a Portuguese sample of incarcerated juvenile offenders. Psychology Crime \& Law, 21(9), 854-870. http://doi.org/10.1080/1068316x.2015.1054386

Pechorro, P., Poiares, C., Barroso, R., Nunes, C., \& Jesus, S. N. (2015). Examining Ethnic Differences in SelfReported Psychopathic Traits Among Portuguese Male Juvenile Offenders. International Journal of Offender Therapy and Comparative Criminology, 59(1), 68-84. http://doi.org/10.1177/0306624x13502942

Pechorro, P., Poiares, C., Marôco, J., \& Vieira, R. X. (2012). Traços psicopáticos e perturbação do comportamento em adolescentes institucionalizados. Psicologia, Saúde E Doenças, 13(2), 399-409.

Pechorro, P., Poiares, C., \& Vieira, R. X. (2008). Caracterização psicológica de uma amostra forense de abusadores sexuais. Análise Psicológica, 4(26), 615-623. http://doi.org/10.14417/ap.520

Pechorro, P., Ray, J. V, Salas-Wright, C. P., Maroco, J., \& Gonçalves, R. A. (2015). Adaptation of the Basic Empathy Scale among a Portuguese sample of incarcerated juvenile offenders. Psychology Crime \& Law, 21(7), 699-714. http://doi.org/10.1080/1068316x.2015.1028546

Penajoia, M. S., Pinho, M. S., \& Dias, I. T. (2015). Influência da familiaridade com procedimentos judiciais de interrogatório na sugestionabilidade interrogativa de reclusos reincidentes. Análise Psicológica, 33(3), 251-264. http://doi.org/10.14417/ap.861

Pimentel, A., Quintas, J., Fonseca, E., \& Serra, A. (2015). Estudo normativo da versão Portuguesa do YLS/CMI - Inventário de avaliação do risco de reincidência e de gestão de caso para jovens. Análise Psicológica, 31(1), 55-71. http://doi.org/10.14417/ap.883

Rebocho, M. F., \& Gonçalves, R. A. (2012). Sexual Predators and Prey: A Comparative Study of the Hunting Behavior of Rapists and Child Molesters. Journal of Interpersonal Violence, 27(14), 2770-2789. http://doi.org/10.1177/0886260512438280

Rebocho, M. F., \& Silva, P. (2014). Target selection in rapists: The role of environmental and contextual factors. Aggression and Violent Behavior, 19(1), 42-49. http://doi.org/10.1016/j.avb.2013.12.003

Rocha, N. B. F., Marques, A. B., Fortuna, R. B., Antunes, A., \& Hoaken, P. N. S. (2014). Effectiveness of cognitive remediation for female inmates: A pilot study. Journal of Forensic Psychiatry and Psychology, 25(2), 224-237. http://doi.org/10.1080/14789949.2014.884617

Saur-Amaral, I. (2011). Towards a Methodology for Literature Reviews in Social Sciences. Investigação E Intervenção Em Recursos Humanos 2011 - Gestão Para a Cidadania. Paper presented at Investigação e Intervenção em Recursos Humanos 2011 - gestão para a cidadania, Porto.

Seruca, T., \& Silva, C. F. (2015). Recidivist criminal behaviour and executive functions: a comparative study. Journal of Forensic Psychiatry \& Psychology, 26(5), 699-717. http://doi.org/10.1080/14789949.2015.1054856

Sharkey, L. (2010). Does Overcrowding in Prisons Exacerbate the Risk of Suicide among Women Prisoners? The Howard Journal of Criminal Justice, 49(2), 111-124. http://doi.org/10.1111/j.14682311.2009.00602.x

Sigre-Leirós, V., Carvalho, J., \& Nobre, P. (2015a). Cognitive schemas and sexual offending: Differences between rapists, pedophilic and nonpedophilic child molesters, and nonsexual offenders. Child Abuse \& Neglect, 40, 81-92. http://doi.org/10.1016/j.chiabu.2014.10.003

Sigre-Leirós, V., Carvalho, J., \& Nobre, P. (2015b). Adult interpersonal features of subtypes of sexual 
offenders. Journal of Forensic and Legal Medicine, 34, 5-10. https://doi.org/10.1016/j.jflm.2015.04.015

Silva, D. R. da, Rijo, D., \& Salekin, R. T. (2012). Child and adolescent psychopathy: A state-of-the-art reflection on the construct and etiological theories. Journal of Criminal Justice, 40(4), 269-277. http://doi.org/10.1016/j.jcrimjus.2012.05.005

Silva, D. R. da, Rijo, D., \& Salekin, R. T. (2015). The evolutionary roots of psychopathy. Aggression and Violent Behavior, 21, 85-96. http://doi.org/10.1016/j.avb.2015.01.006

Stack, S. J., \& Tsoudis, O. (1997). Suicide risk among correctional officers: A logistic regression analysis. Archives of Suicide Research, 3(3), 183-186. http://doi.org/10.1023/A:1009677102357

Stewart, E. C. (2007). The Sexual Health and Behaviour of Male Prisoners: The Need for Research. The Howard Journal of Criminal Justice, 46(1), 43-59. http://doi.org/10.1111/j.1468-2311.2007.00453.x

Talina, M., Thomas, S., Cardoso, A., Aguiar, P., Caldas de Almeida, J. M., \& Xavier, M. (2013). CANFOR Portuguese version: validation study. BMC Psychiatry, 13(1), 157. http://doi.org/10.1186/1471244X-13-157

Tavares, S., Caetano, A., \& Silva, S. (2007). Não há bela sem senão: A identificação organizacional, os comportamentos de dedicação ao trabalho e o conflito trabalho-família. Psicologia, 21, 133-149.

Triplett, R., Mullings, J. L., \& Scarborough, K. E. (1999). Examining the effect of work-home conflict on work-related stress among correctional officers. Journal of Criminal Justice, 27(4), 371-385. http://doi.org/10.1016/S0047-2352(98)00066-X

Trujillo, H. M., Jordán, J., Antonio Gutiérrez, J., \& González-Cabrera, J. (2009). Radicalization in Prisons? Field Research in 25 Spanish Prisons. Terrorism and Political Violence, 21(4), 558-579. http://doi.org/10.1080/09546550903153134

Useem, B., \& Clayton, O. (2009). Radicalization of U.S. prisoners. Criminology \& Public Policy, 8(3), 561592. http://doi.org/10.1111/j.1745-9133.2009.00574.x

Vilariño, M., Amado, B. G., \& Alves, C. (2013). Menores infractores: un estudio de campo de los factores de riesgo. Anuario de Psicología Jurídica, 23(1), 39-45. http://doi.org/10.5093/aj2013a7

Wooldredge, J., Griffin, T., \& Pratt, T. (2001). Considering hierarchical models for research on inmate behavior: Predicting misconduct with multilevel data. Justice Quarterly, 18(1), 203-231. http://doi.org/10.1080/07418820100094871

\section{ANEXO I}

Critérios de inclusão: Artigos que abordam o contexto prisional na relação com variáveis do domínio da psicologia. Motivo: na relação com os objetivos do estudo, a revisão sistemática apresentada inclui apenas artigos que, independentemente da sua natureza, privilegiem variáveis psicológicas.

Critérios de exclusão: Artigos empíricos que não recolhem dados em contexto português. Motivo: ao nível dos estudos empíricos, o presente trabalho centra-se exclusivamente em investigação com recolha de dados em contexto prisional português.

Historial do artigo

Recebido 23/09/2016

Aceite 28/11/2016

Publicado $\quad 07 / 2017$ 